\title{
P 240 AUDIT OF PALLIATIVE CARE PATIENT IDENTIFICATION IN GENERAL PRACTICE
}

Richard Courtney, ${ }^{1}$ Alan Calow ${ }^{2} .{ }^{1}$ Manchester Medical School, Blackburn, England;

${ }^{2}$ Redlam Medical Practice, 62-64 Redlam, Blackburn, Lancashire.

\subsection{6/bmjspcare-2014-000654.281}

Background The Gold Standards Framework highlights the need for early identification of palliative care patients within the community. Thus allowing optimization of patient care and avoidance of unnecessary treatments near the end of life. The Dying Matters website states GP palliative registers should only have approximately $25 \%$ of cancer diagnoses on them.

Aim To assess if the practice wad correctly registering palliative care patients and what proportion of the palliative register had cancer diagnoses.

Method Using the GP computer system (Emis) a search was made of the most recent deaths over 7 months. This was cross referenced with the palliative care register at that time to find which patients had not been correctly registered onto the palliative care register prior to their deaths. The notes of these non-palliative care registered deaths were assessed retrospectively to see if the could have been previously added onto the palliative register. Markers within the medical records were previous patient/relative discussion around palliative care, DNAR forms and the use of Prognostic Indicators Guidance.

Results A total of 33 patient deaths were identified over 7 months. 12 were correctly palliative care registered. 11 deaths could not have been predicted from the records and 10 deaths were expected and therefore eligible for the palliative care register.

Notably $66 \%$ of the palliative list had a cancer diagnosis, whereas 9 out of 10 of the unregistered but expected deaths had non-cancer diagnoses. This highlighted clearly that the other common palliative groups were being missed.

Conclusion Performing such an audit can allow any practice to evaluate their concordance with the current Gold Standards Framework. It re-iterated how the Prognostic Indicator Guidance could be used to identify non-cancer palliative care patients for inclusion onto the palliative register. 